\title{
Percepción de las personas adultas mayores sobre los servicios de la Fiscalía de la Tercera Edad
}

Rafaela Esmeralda Méndez Montoya ${ }^{1}$

Lourdes Valladares Zavala²

\section{RESUMEN}

El presente estudio describe la percepción de las personas adultas mayores sobre los servicios que brinda la Fiscalía de la Tercera Edad de Tegucigalpa, la cual tiene importancia debido al aumento creciente de esta población a nivel nacional y mundial. El interés por la temática surgió del contacto con las personas adultas mayores, como trabajadora social de la Dirección de Medicina Forense, víctimas de violencia intrafamiliar y del abandono, razones que los han llevado a acudir a dichas instancias legales en defensa de sus derechos.

El artículo presenta un análisis del contexto en el cual se desarrolló la investigación, haciendo referencia a la problemática de maltrato que sufre la población desde el enfoque de derechos humanos, ya que para comprender e intervenir en la problemática actual de las personas adultas mayores es necesaria una nueva concepción de la vejez, desde este enfoque y del trabajo conjunto de profesionales, familias, comunidad y Estado, constituidos como agentes de apoyo social para promover una vejez con seguridad, bienestar y ejercicio de ciudadanía.

Este estudio tuvo un enfoque cualitativo, basado en el método fenomenológico, ya que su propósito fue describir cómo las personas adultas mayores percibían los servicios que brinda la Fiscalía de la Tercera Edad y de acuerdo a estos resultados, se describe y caracteriza el fenómeno. Para recoger la información se contó con la colaboración de personas adultas mayores que hicieron uso de los servicios de la Fiscalía durante el I semestre de 2013.

\footnotetext{
${ }^{1}$ Beneficiaria de una beca de estudiante de posgrado y estudiante de la Maestría Latinoamericana en Trabajo Social y Gestión del Desarrollo, Facultad de Ciencias Sociales, UNAH: esmendez16@hotmail.com

${ }^{2}$ Asesora, Facultad de Ciencias Sociales, UNAH: valladareslourdes@yahoo.com
} 
El desarrollo de la investigación contempló, para la producción, de datos cualitativos, la técnica de la entrevista semiestructurada y se contó con un universo de 67 personas adultas mayores, de las cuales 20 fueron hombres y 47 mujeres. En cuanto a los hallazgos es importante decir que la población adulta mayor que formó parte de la muestra de este estudio, se siente satisfecha con la labor que realiza esta institución, ya que el usuario está contento cuando los servicios cubren o exceden sus expectativas; pero, si estas son bajas o si el usuario tiene acceso limitado a cualquiera de los servicios, puede ser que esté insatisfecho con recibir servicios relativamente deficientes.

Palabras claves: personas adultas mayores, Fiscalía de la Tercera Edad, violencia intrafamiliar, abandono, satisfacción de servicios.

\section{ABSTRACT}

The present study describes the perception of older people about the services provided by the Office for third age attention in Tegucigalpa, which is important due to their increased population, globally and nationwide. As a social worker from the Department of Forensic Medicine, interest in the subject arose from contact with older adults, which were victims of domestic violence and neglect, and therefore led them to resort to such legal remedies to defend their rights.

The article presents an analysis of the context in which the research was conducted, referring to the problem of abuse suffered by the population from the human rights perspective. In order to understand the current elderly problems, a new conception of old age is needed, to understand this perspective and the joint work of professionals, families, community and state, constituted as agents of social support to promote old age security, welfare and citizenship.

This study used a qualitative approach, based on the phenomenological method, since its purpose was to describe how the elderly perceive the services provided by the Office for third age attention. According to these results, the phenomenon was described and characterized. The information was collected by the elderly using the Office's services during the first half of 2013.

For production work qualitative data, the research development contemplated the technique of semi-structured interview. 67 older adults were interviewed, of which 20 
were men and 47 were women. As for the findings it is important to say that older adults who took part in the study sample, were satisfied with the work of this institution. They also expressed their satisfaction with the coverage of services and for some, it exceed their expectations. However, some users might have expressed low expectations, because they had limited access to the services provided by the Office.

Keywords: older persons, Senior Attorney, domestic violence, abandonment, service satisfaction. 


\section{INTRODUCCIÓN}

La violencia intrafamiliar y el abandono de los ancianos está aumentando a medida que la población envejece y cambia la dinámica social. La OMS (2002) calcula que entre un $4 \%$ y un $6 \%$ de las personas mayores han sufrido alguna forma de maltrato: físico, psicológico, emocional, financiero o debido a negligencias.

En Honduras, a partir de 1997, se creó la Fiscalía Especial de Protección al Consumidor y la Tercera Edad con el propósito de representar, defender y proteger los derechos de las personas adultas mayores, la cual tiene como objetivo primordial el cumplimiento o sanción para todas aquellas personas que infrinjan la Ley Integral de Protección al Adulto Mayor y Jubilados, que es la actual ley vigente en nuestro país a favor de este sector poblacional.

Se sabe que la violencia intrafamiliar y el abandono es una clara violación a los derechos humanos y las personas adultas mayores son víctimas de este tipo de delitos, por eso se les atiende en la Fiscalía. Asimismo, se considera para el análisis y lectura de datos, la estrategia propuesta por Sandra Huenchuan de la CELADE (2009), porque a nivel de América Latina es experta en la temática de envejecimiento, esta incluye el desarrollo y la aplicación de conceptos y contenidos particulares con base a tres dimensiones: normativa, procesal y contenidos.

Es así que el presente estudio se realizó desde la Fiscalía de la Tercera Edad con el propósito de dar respuesta a la pregunta de investigación: ¿Cuál es la percepción de las personas adultas mayores sobre los servicios que brinda la Fiscalía de la Tercera Edad de la ciudad de Tegucigalpa?

Como aporte social se espera que los hallazgos y recomendaciones de esta investigación sirvan para mejorar la calidad de asistencia por parte de esta institución encargada de velar por el bienestar de las personas adultas mayores, ya que constantemente este sector demanda el respeto a sus derechos y condiciones dignas de vida, así como también exigen a los Gobiernos de turno políticas públicas y programas que los beneficien.

El estudio también tiene un aporte académico, el cual consiste en la generación de conocimientos teóricos e información sobre la temática, la cual puede ser de apoyo para futuras investigaciones relacionadas con el maltrato a personas adultas mayores, dado que en nuestro país, de cierta manera, esta problemática ha sido margina- 
da y los estudios en torno a esta temática son escasos, por eso la importancia de esta investigación.

\section{METODOLOGÍA}

Este estudio tiene un enfoque cualitativo basado en el método fenomenológico, ya que su propósito es describir cómo las personas adultas mayores perciben los servicios que brinda la Fiscalía de la Tercera Edad y de acuerdo a estos resultados se describe y caracteriza el fenómeno.

Para recoger la información de este estudio se contó con la colaboración de personas adultas mayores que hicieron uso de los servicios de la Fiscalía durante el I semestre de 2013, es por eso que tiene una temporalidad transversal, porque se estudió el fenómeno en un momento dado.

Para la selección de la muestra se hizo una revisión de la base de datos y expedientes, estos se clasificaron según sexo, el tipo de servicios recibido y avance del proceso. De acuerdo a esta revisión se contó con un universo de 67 personas adultas mayores, de las cuales 20 son hombres y 47 mujeres; 20 son denuncias por el delito de abandono y 47 por violencia intrafamiliar.

La muestra fue no probalística y se constituyó con dos personas adultas mayores que denunciaron violencia intrafamiliar del sexo masculino y dos del sexo femenino, dos personas adultas mayores que reportaron abandono del sexo masculino y dos del sexo femenino, dos personas adultas mayores que su caso haya estado en proceso y dos que su caso haya sido concluido, para hacer un total de 12 entrevistas.

El tipo de muestreo utilizado fue intencional, ya que la selección de las unidades de análisis se hizo basándose en los siguientes criterios: personas adultas mayores de 60 años, haber presentado denuncia en la Fiscalía durante el I semestre de 2013, sexo, tipo de servicio utilizado, delito denunciado y avance del caso o proceso.

Una vez que fue seleccionada la muestra se aplicó la técnica de recolección de información prevista para este estudio, que fue la entrevista semiestructurada de acuerdo a categorías definidas en el marco teórico y categorización de variables. Para el análisis de los datos se elaboró una matriz en Excel para facilitar la lectura de los hallazgos. 


\section{ANÁLISIS}

Para el desarrollo de este estudio fue importante conocer algunas características demográficas de la población adulta mayor de nuestro país, así como aspectos de la situación real que presentan este sector poblacional.

\section{Marco contextual}

El envejecimiento es un fenómeno ligado a la llamada transición demográfica que se evidencia en la declinación de las tasas de mortalidad y fecundidad y sus efectos en la estructura de la población (Zelaya Ochoa, 2012). Algunos elementos como el acceso a los servicios de salud, adelantos de la medicina, alto nivel educativo y saneamiento ambiental, son factores que están relacionados con el aumento de la esperanza de vida e incide, a su vez, en el envejecimiento gradual de la población.

En la pirámide poblacional de Honduras en 2010, se evidencia que el crecimiento de la población mayor alcanzó el 5.9 \%; se proyecta que para el año 2030 llegará a un 10 \% y en el año 2050 se incrementará hasta el 17.4 \% (Flores Fonseca, 2007).

Según el Instituto Nacional de Estadísticas en el año 2010 la población hondureña era de 8,045,990 personas, de las cuales 789,871 eran adultas de 60 años en adelante. En la actualidad este sector representa el $8.5 \%$ del total de la población hondureña.

En Honduras, país en vías de desarrollo considerado como uno de los países con economías en transición, la mayoría de las personas que ahora son adultas mayores participan en la economía informal, lo que suele privarlos de las ventajas del trabajo regular.

Al analizar la relación de los ingresos con la edad del jefe del hogar, los datos de la encuesta permanente de hogares de propósitos múltiples (2010) muestra que más de dos tercios de la población adulta mayor en Honduras declaró ser jefe de hogar; pero al desagregar los datos según sexo, son más los hombres adultos mayores que tienen esta condición.

En el 2001, $64.7 \%$ de los adultos mayores declaró ser jefe de hogar, para los años 2005 y 2010 se refleja una leve disminución de $63.8 \%$ y $63.7 \%$, respectivamente (Zelaya Ochoa, 2012). La posición de los adultos mayores en la población activa 
actualmente incluye con frecuencia salarios más bajos y falta de progresión laboral a causa de historiales de trabajos interrumpidos, obligaciones de asistencia a la familia y mayor dificultad para encontrar trabajo después de perder el empleo.

En cuanto a las características educativas de la población adulta mayor en Honduras, los datos reflejan que un porcentaje muy alto de la población adulta mayor era analfabeta, se observa que en el 2001 este porcentaje corresponde al $49.7 \%$, para el 2010 presenta una disminución de 6.4 puntos porcentuales, esto probablemente esté relacionado con la implementación de diversos programas de alfabetización que se han desarrollado tanto a nivel nacional como local (Zelaya Ochoa, 2012).

Al desagregar los datos según sexo, se observa que existe predominio de mujeres analfabetas con respecto a los hombres, situación que está directamente relacionada con factores culturales, puesto que se creía que las mujeres solamente debían estar formadas para las labores domésticas y ser buenas esposas y madres. Los datos reflejan un comportamiento muy similar durante los años 2001, 2005 y 2010 (Zelaya Ochoa, 2012).

Las personas adultas mayores en Honduras se encuentran entre los grupos poblacionales con menor escolaridad, debido a diversos factores entre los que destacan: las escasas oportunidades educativas debido a la baja cobertura del sistema educativo cuando este grupo estaba en edad escolar, las condiciones de pobreza, la incorporación temprana al mercado laboral, la poca importancia que se le daba años atrás a la formación académica, entre otras (Zelaya Ochoa, 2012).

Este sector de la población habrá de requerir mayor atención debido a que, por efecto de la transición demográfica, el número de personas adultas mayores habrá de incrementarse a casi un millón de habitantes en 2025. También se habrá alcanzado una mayor esperanza de vida al nacer y se habrán disminuido los índices de mortalidad y natalidad. Asimismo, la población en edad productiva (15 a 64 años de edad) disminuirá paulatinamente en términos relativos (ONU, 2002).

En un diagnóstico publicado por la Asociación Nacional de los Adultos Mayores de Honduras, en la web de Pensionados y Jubilados Catrachos (2014), se presentan datos importantes acerca de la situación real que viven las personas adultas mayores en nuestro país. De los hogares encuestados, un $5.0 \%$ tiene el beneficio de una jubilación o pensión mensual, con el agravante de que en la gran mayoría de los casos son jubilaciones o pensiones de mera subsistencia; pero, existe un $6.0 \%$ de esta población que a través de una vida de ahorro y sacrificio ha logrado concretar un 
patrimonio propio, lo que le permitirá vivir con decoro y dignidad el resto de su existencia sin tener que depender del Estado o de sus familiares.

También hay un $9.0 \%$ de adultos mayores que se cree (sin tener las evidencias del caso) que están siendo atendidos por su propia familia. Con los datos anteriores se puede decir que se encuentra apenas un $20 \%$ de adultos mayores que tiene un beneficio por jubilación o pensión, cuenta con patrimonio propio o está siendo atendido por sus familiares y el $80 \%$ de la población mayor de Honduras, que es un aproximado de quinientos sesenta mil $(560,000)$ compatriotas, subsiste en pobreza y en extrema pobreza, sin esperanza alguna de poder revertir ese estado sombrío, por eso se vuelve necesaria la voluntad política bienhechora del Estado a través de los Gobiernos de turno para mejorar dichas condiciones.

\section{Caracterización de los servicios que brinda la Fiscalía de la Tercera Edad}

La Fiscalía Especial de Protección del Consumidor y la Tercera Edad fue creada en 1997 y es la dependencia del Ministerio Público encargada de representar, defender y proteger los derechos de los consumidores y personas de la tercera edad, aplicando el procedimiento penal cuando los efectos de la acción de consumo de un bien 0 servicio hayan afectado la salud, la buena fe, la economía u otros bienes jurídicos por el ordenamiento jurídico nacional en la materia de consumidor y tercera edad, cuando se supere el procedimiento administrativo previsto en la ley.

Para este estudio se hará referencia a la labor que realiza esta Fiscalía solo con personas de la tercera edad (adulto mayor), cuya tarea fundamental es el ejercicio de la acción penal pública en la atención de la protección del adulto mayor. En tal sentido, conoce e investiga aquellas denuncias que por incumplimiento de la Ley Integral de Protección al Adulto Mayor y Jubilados y por las conductas ilícitas cometidos en contra de ellos tipificadas en el Código Penal. Fue creada como una iniciativa del Estado para el cumplimiento de la Ley Integral de Protección al Adulto Mayor y Jubilados. La Fiscalía de la Tercera Edad atiende específicamente denuncias por los siguientes delitos:

1. Violencia Intrafamiliar: vista como el maltrato a la persona adulta mayor por parte de sus propios familiares o personas con las que conviven, es decir, hijos y nietos, por este delito se otorgan penas que van de $1 \mathrm{a} 3$ años.

2. Abandono: se trata cuando la familia o convivientes de una persona adulta mayor ya no quieren tenerlos porque lo ven como un estorbo; por lo general esta idea 
se da por falta de dinero para mantener al adulto mayor, como al resto de los familiares o cohabitantes del hogar, 0 en ocasiones por la falta de sensibilidad. Por este delito se otorgan penas que van de 1 a 3 años y de 3 a 6 años.

La Fiscalía ampara sus acciones dentro del siguiente marco legal: la Constitución de la República, la Ley Integral de Protección del Adulto Mayor y Jubilados, Código Penal, Código Procesal Penal, entre otras. Esta Fiscalía con sus acciones pretende tener efectos en cuanto al bienestar del adulto mayor, la prisión a agresores, lograr sentencias condenatorias y el aumento en las denuncias.

Es a partir de este contexto que se pretendió conocer cómo las personas adultas mayores que han hecho denuncias en la Fiscalía, perciben los servicios brindados, ya que según estadísticas del Ministerio Público anualmente el $25 \%$ del total de las denuncias recibidas son por violencia y maltrato hacia adultos mayores. Estas denuncias tienen que ver, específicamente, con abandono y pleitos en el núcleo familiar, en el que se incluye la violencia psicológica y física que a veces, hasta por varios años, han venido soportando los familiares en edad adulta.

Lo anterior se refleja en los datos proporcionados por la Unidad Nacional de Organización y Depuración de Expedientes del Ministerio Público, en donde se muestra la criminalidad denunciada en el 2010 en la Fiscalía de la Tercera Edad de Tegucigalpa con 117denuncias (15 por abandono y 102 por violencia intrafamiliar), en el 2011 se registran 202 denuncias (17 por abandono y 185 por violencia intrafamiliar) y en el año 2012 se registraron 183 denuncias (24 por abandono y 159 por violencia intrafamiliar).

Estos fenómenos no dejan de ser una preocupación para las instituciones que trabajan para este sector poblacional, ya que la violencia intrafamiliar y el abandono es un problema de violación de derechos humanos, al cual están siendo sometidas estas personas que acuden a la Fiscalía y cada año las estadísticas reflejan que hay un incremento, por tanto, en eso radica la preocupación sobre el tema y la necesidad de este estudio.

Dentro de los logros por parte de esta Fiscalía, durante el 2012, están que de 223 denuncias: un $64 \%$ de los casos (aproximadamente 200) se dio por cerrado; esto corresponden a archivos administrativos en donde la Fiscalía de Protección de la Tercera Edad incidió para que al ciudadano se le resolviera satisfactoriamente su demanda. En algunos casos los mismos ofendidos pidieron la no continuación de la denuncia porque llegaron a acuerdos con los familiares o personas que están a cargo 
del adulto mayor que había denunciado, por lo que estos casos pasaron a lo que ellos llaman cierre administrativo.

En un $5 \%$ de los casos se dieron órdenes de captura, en un $4 \%$ se establecieron criterios de oportunidad, en un $8 \%$ se lograron autos de prisión, los requerimientos fiscales se dieron en un $18 \%$ y solo en un $1 \%$ se lograron sentencias.

Con este estudio se quiere tomar conciencia y empoderamiento de la problemática de las personas adultas mayores, para buscar acciones que favorezcan su bienestar y condiciones de vida de este sector generacional, ya que los servicios que brinda la Fiscalía forman parte de las acciones del Estado para garantizar el cumplimiento de sus derechos humanos.

\section{Marco conceptual}

Este estudio se orienta con el enfoque de derechos humanos, ya que este paradigma propone que las personas adultas mayores deben ser consideradas sujeto de derecho y no un objeto de asistencia y cuidado.

Mariela Ramírez, en su estudio sobre Calidad de vida en la tercera edad, habla de un nuevo paradigma y este es la aplicación del enfoque de derecho a las políticas públicas:

Esta mirada a la vejez promueve el empoderamiento de los adultos mayores y, consecuentemente, una sociedad integrada, desde el punto de vista de las edades cronológicas de los habitantes... manifestar que las personas mayores son sujetos de derecho y no solamente objetos de protección, significa que disfrutan de garantías, pero también tienen responsabilidades respecto de sí mismos, su familia y la sociedad... Los derechos humanos son universales, civiles (libertad de pensamiento, igualdad ante la ley y propiedad), políticos (derecho al sufragio y libertad de expresión), sociales (trabajo, sindicalización, huelga, salud, educación, protección familiar, vivienda)... Toda persona tiene derecho a un nivel de vida adecuado que le asegure, así como a su familia, la salud y el bienestar, y en especial la alimentación, el vestido, la vivienda, la asistencia médica y los servicios sociales necesarios; enfermedad, invalidez, viudez, vejez y otros casos de pérdida de sus medios de subsistencia por circunstancias independientes de su voluntad (2008, p.58-59).

Los derechos sociales están amparados por la Constitución de la República, sin embargo, se dan situaciones cotidianas en donde los derechos se ven vulnerados, como el caso de las personas adultas mayores que son las unidades de análisis de 
este estudio, las cuales han recurrido a los servicios de la Fiscalía de la Tercera Edad porque les están siendo violentados sus derechos al ser víctimas de violencia intrafamiliar 0 abandono por parte de los familiares, cuando son los encargados de dar la contención necesaria para el disfrute pleno de las personas en esta etapa de la vida.

El enfoque de derechos propicia la titularidad de derechos humanos de todos los grupos sociales y, de ese modo, contribuye a que quienes en el pasado fueron excluidos, en el presente sean tratados sobre la base de la igualdad y el respeto de la dignidad humana para favorecer la integración social y, con ello, la construcción de una "sociedad para todos" (Naciones Unidas, 1995; Huenchuan, 2009).

Desde este ángulo, uno de los desafíos centrales de las políticas públicas basadas en los derechos es determinar la forma de contribuir a la construcción de una sociedad con cabida para todos, en donde las personas, con independencia de su edad o de otras diferencias sociales, tengan las mismas oportunidades para hacer efectivo el respeto y ejercicio pleno de sus derechos humanos y libertades fundamentales (Huenchuan y Rodríguez, 2010).

Para este estudio se consideró el planteamiento de Sandra Huenchuan Huenchuan (2009), porque es experta en la temática de envejecimiento con enfoque de derechos humanos del Centro Latinoamericano y Caribeño de Demografía y la CEPAL. La estrategia que ella propone permite tener una idea más amplia de la labor que realiza la Fiscalía de la Tercera Edad con base al análisis de la dimensión del contenido, normativa y procesal, ya que si solo se hubiese considerado la variable satisfacción de servicios, los resultados iban a quedar cortos y sin dar respuesta a la realidad del fenómeno de estudio.

Según Huenchuan (2009), la estrategia para promover el enfoque de los derechos humanos en el abordaje de los asuntos de las personas mayores debe incluir el desarrollo y la aplicación de conceptos y contenidos particulares basados en tres dimensiones: normativa, procesal y de contenidos, de manera que sirva para avanzar hacia el ejercicio de los derechos ciudadanos por parte de todos los grupos sociales:

1. Dimensión de contenidos: este abordaje del enfoque de los derechos aplicados a los asuntos del envejecimiento supone dos debates asociados. En primer lugar se ubican aquellas acciones concretas de aplicación y de responsabilidad que se ponen en práctica mediante programas sectoriales o específicos dirigidos exclusivamente a las personas mayores (targeting), considerando que su orientación y sus prestaciones permitan avanzar en el cumplimiento de los derechos en la 
vejez. Como características básicas, estos programas deben ser universales y contar con mecanismos para hacer exigibles esas prestaciones y servicios (CEPAL, 2006 y Huenchuan, 2011). En segundo lugar está la aplicación transversal de una perspectiva amigable para las personas mayores en el marco de la corriente general de las políticas públicas (maestreaming), lo que con lleva la creación y el desarrollo de mecanismos de cumplimiento de las obligaciones por parte del Estado dentro de su quehacer general.

2. Dimensión normativa: la aplicación del enfoque de los derechos humanos en los asuntos de las personas mayores implica que las acciones públicas y las instituciones encargadas de su atención deben basarse explícitamente en las normas internacionales sobre derechos humanos, puesto que, independientemente de que su situación no esté explícitamente incorporada, los instrumentos internacionales y regionales de derechos humanos de orden vinculante pueden brindar una protección jurídica de los derechos de las personas mayores si son empleados de manera adecuada.

3. Dimensión procesal: incorporar el enfoque de derechos a los asuntos de las personas mayores supone que la legislación y las políticas sobre envejecimiento deben promover y proteger los derechos y libertades fundamentales en la vejez. Para que esto se concrete, se requiere que los poderes políticos establezcan los medios y los recursos con que se harán efectivos, como por ejemplo la constitucionalización de derechos, la creación de leyes especiales de protección y la puesta en marcha de las políticas o planes de acción. Asimismo, es necesario asignar presupuestos que financien la puesta en práctica de las disposiciones normativas y políticas a las que un Estado se obliga. Todo ello con base a tres criterios básicos: la no discriminación, la progresividad y la participación.

Con lo anterior se aprecia que el uso de la normativa internacional de derechos humanos es una herramienta esencial para promocionar y proteger positiva y ampliamente los derechos en la vejez. Fundamentalmente, permite traspasar la esfera individual, establecer responsabilidades para la sociedad en general y para los Gobiernos, junto con concentrar los esfuerzos para que los sujetos controlen de manera independiente sus vidas (Huenchuan, 2009).

El enfoque de derechos reivindica el papel del Estado para garantizar el desarrollo humano y establece la política social como un derecho social, contempla el impulso de políticas institucionales tendientes a que las personas se apropien de sus dere- 
chos y participen de manera activa en su desarrollo social y controlen las acciones públicas en esa materia (Solís, 2003).

Partiendo de este enfoque de derechos en donde el papel del Estado es impulsar políticas institucionales para que las personas se apropien de sus derechos, es que se centra este estudio, teniendo como unidades de análisis las personas adultas mayores que acuden a la Fiscalía de la Tercera Edad con base a que sus derechos están siendo violentados y, en este sentido, acuden a dicha institución como ente encargado de velar por el respeto de los mismos.

\section{CONCLUSIONES}

1. La Fiscalía de la Tercera Edad cumple sus funciones amparada en la estrategia de derechos humanos promovida por la Sandra Huenchuan, ya que sus acciones están sustentadas en lineamientos de normas a nivel nacional e internacional con el propósito de velar por el bienestar de la población adulta mayor hondureña, también para defender sus derechos porque son personas víctimas de maltrato.

2. La labor que realiza la Fiscalía de la Tercera Edad, en cuanto a la parte procesal, se ampara en leyes nacionales como el Código Procesal Penal y la Ley Integral de Protección al Adulto Mayor y Jubilados.

3. Es importante decir que la población adulta mayor que formó parte de la muestra de este estudio se siente complacida con la labor que realiza esta institución, ya el usuario está satisfecho cuando los servicios cubren o exceden sus expectativas; si estas son bajas o si el usuario tiene acceso limitado a cualquiera de los servicios, puede ser que esté insatisfecho con recibir servicios relativamente deficientes.

4. En cuanto a las limitaciones o dificultades que las personas adultas mayores encontraron al momento de hacer uso de la Fiscalía de la Tercera Edad, está el acceso para llegar a dichas instalaciones, por tanto, es una de las consideraciones a tomar en cuenta para dar una mayor atención a este sector poblacional. 


\section{AGRADECIMIENTOS}

A la Dirección de Investigación Científica y Posgrado de la UNAH por el financiamiento otorgado para la realización del presente estudio. También a Corina Hernández y Lourdes Valladares por su apoyo y orientación durante este proceso de enseñanza-aprendizaje en el Posgrado Latinoamericano de Trabajo Social de la UNAH. Igualmente, gracias a Sara Sagastume, coordinadora de la Fiscalía de la Tercera Edad, por permitir realizar el estudio desde esta institución.

\section{BIBLIOGRAFÍA}

Adams, Y. (2011). Maltrato en el adulto mayor institucionalizado. Realidad e invisibilidad. Revista Médico Clínico Condes, 23(1), 84-90. Chile.

CEPAL. (2006). La protección social de cara al futuro: acceso, financiamiento y solidaridad. Naciones Unidas.

CEPAL. (2007). Familias y políticas públicas en América Latina: Una historia de desencuentros. Santiago de Chile: Naciones Unidas.

Flores Fonseca, M. A. (2007). La transición demográfica de Honduras. Tegucigalpa: Fondo de Población de las Naciones Unidas.

IUDPAS-UNAH. (2013). Boletín sobre muertes violentas y femicidios. Tegucigalpa.

IUDPAS-UNAH. (2014). Violencia contra las mujeres. Boletín Especial del Observatorio de la Violencia, 17. Tegucigalpa.

IUDPAS-UNAH. (2013). Mortalidad y otros. Boletín Especial del Observatorio de la Violencia, 28. Tegucigalpa.

Pensionados y Jubilados Catrachos. (2014). Aumenta la demanda de servicios para adultos mayores en Honduras. Recuperado de: http://jubilados-y-pensionados-catrachos.globered.com/categoria.asp?idcat=359

Huenchuan, S. (2009). Envejecimiento, derechos humanos y políticas públicas. Santiago de Chile: CEPAL.

Huenchuan, S. (2011). Los derechos de las personas mayores. Hacia un cambio de paradigma. Santiago de Chile: CELADE-CEPAL.

Huenchuan, S. y Rodríguez, L. (2010). Envejecimiento y derechos humanos: Situación y perspectiva de protección. Santiago de Chile: CEPAL.

INE. (2010). XLIV encuesta permanente de hogares de propósitos múltiples. Tegucigalpa. 
INE. (2013). XLIV encuesta permanente de hogares de propósitos múltiples. Tegucigalpa.

Ley Integral de Protección al Adulto Mayor y Jubilados. (2007). La Gaceta No. 31,361. Tegucigalpa.

Organización Mundial de la Salud. (2002). Declaración de Toronto para la prevención global del maltrato a personas mayores. Ginebra.

Organización de las Naciones Unidas. (1995). Informe Argentino sobre Desarrollo Humano. Argentina.

Organización de las Naciones Unidas. (2002). Informe sobre Desarrollo Humano. New York, Estados Unidos.

Ramírez, M. (2008). Calidad de vida en la tercera edad. Una población subestimada por ellos y por su entorno. Tesis. Universidad Nacional de Cuyo. Argentina.

Solís, R. (2003). La definición de políticas públicas. Revista Dossier, (5), 47-52.

Zelaya Ochoa, Marysabel. (2012). El envejecimiento en Honduras: una característica sociodemográfica del adulto mayor. Revista Población y Desarrollo: Argonautas y Caminantes, (8), 83-93. Tegucigalpa. 